\title{
Interference Cancellation Method without Feedback Amount for Three Users Interference Channel
}

\author{
Xinji Tian, Haotian Zhang, Wenjie Jia \\ School of Physics \& Electronic Information Engineering, Henan Polytechnic University, Jiaozuo, China \\ Email: tian215216@sohu.com
}

How to cite this paper: Tian, X.J., Zhang, H.T. and Jia, W.J. (2017) Interference Cancellation Method without Feedback Amount for Three Users Interference Channel. Open Access Library Journal, 4: e3574.

https://doi.org/10.4236/oalib.1103574

Received: March 30, 2017

Accepted: May 1, 2017

Published: May 4, 2017

Copyright $\odot 2017$ by authors and Open Access Library Inc.

This work is licensed under the Creative Commons Attribution International License (CC BY 4.0).

http://creativecommons.org/licenses/by/4.0/

\begin{abstract}
In this paper, we investigate how to cancel multi-user interference without feedback amount over multiple-input multiple-output (MIMO) interference channel using space-time code. Three transmitter-receiver pairs are considered and each node is equipped with four antennas. Space-time codeword with dimension $4 \times 4$ is designed for three transmitters, respectively. The number of interfering codeword is reduced through the design of codeword. Then, multi-user interference is cancelled by a linear operation on the received signals. Compared with the existing scheme for the same scene, the transmission efficiency is improved, and the unidirectional cooperative link is not required. Simulation results demonstrate the validity of theoretical analysis.
\end{abstract}

\section{Subject Areas}

Information and Communication Theory and Algorithms

\section{Keywords}

Interference Channel, Space-Time Codeword, Multi-User Interference, Interference Cancellation, Transmission Efficiency

\section{Introduction}

Reliability and effectiveness are important factors for wireless communications. Multiple-input multiple-output (MIMO) systems have the ability to improve both and hence, are immensely popular. In theory, MIMO systems can achieve extremely high spectral efficiency by transmitting independent data stream simultaneously. However, multiple users, transmitting different codewords to multiple receivers simultaneously using the same band, are not able to collaborate with each other [1]. There is serious interference at each receiver, and the 
effectiveness becomes limited. Therefore, it is quite important to design transmission techniques to eliminate multi-user interference [2].

Several kinds of interference cancellation methods have been conducted, such as pre-coding at the transmitters, multi-user detection of the receivers, and interference alignment [3] [4]. Interference alignment methods align the interference caused at each receiver from all the transmitters along the same direction while the desired signals occupy linearly independent signal dimensions. In this case, the desired signals can be decoded free from interference by zero-forcing algorithm.

A popular way to approximating the sum-capacity for interference alignment is using the concept of degrees of freedom (DoF), in terms of transmission efficiency. Many works have been conducted to evaluate DoF [5] [6]. However, effectiveness is contract to reliability in communication systems. With high DoF, reliability in terms of the diversity gain is needed to be improved. Therefore, it is meaningful to combine interference alignment and space-time code, which can offer diversity gain [7] [8]. [9] proposes a new interference alignment scheme using Alamouti code for two users MIMO X channel, in which zero vector is introduced into each codeword. The diversity gain of [9] is 2 , which is brought by Alamouti code. Space-time codeword with dimension $4 \times 4$ is designed and zero vectors are introduced into each codeword over two users MIMO $\mathrm{X}$ channel with four antennas at each user, in which the diversity gain is 4 [10]. The multiuser interference is mitigated through interference alignment and linear operation on the received signals. Motivated by [9], interference alignment and Alamouti code are introduced into MIMO interference channel with three transmitter- receiver pairs [11]. The multi-user interference is mitigated through interference alignment and unidirectional cooperative link. However, channel state information (CSI) is required at the transmitters. It is very difficult to estimate the CSI in a mobility environment. Therefore, it is crucial to consider transmission scheme combined interference alignment and space-time code without CSI.

Under this motivation, interference cancellation method without feedback is proposed for MIMO interference channel with three transmitter-receiver pairs where each node is equipped with four antennas. Space-time codeword with dimension $4 \times 4$ is designed for three transmitters, respectively. Each codeword includes eight independent modulated symbols and zero matrices are introduced into each codeword, through which the number of interfering codeword is reduced. Then, multi-user interference is cancelled by a linear operation on the received signals. The transmission efficiency of the proposed scheme is higher than that of [11]. Theoretical analysis shows that the diversity of the proposed scheme is the same as that of [11].

Notation: The operators $(\cdot)^{*}$ and $(\cdot)^{H}$ stand for complex conjugation and conjugation transpose, respectively. $\|\cdot\|$ denotes Frobenius norm of the enclosed term. $E(\cdot)$ is the expectation operations. 


\section{System Model}

The system model is shown in Figure 1. There are three dedicated transmitterreceiver pairs, where each transmitter has an individual message to be communicated to its respectively receiver. All the transmitters and receivers are assumed to have four antennas. We assume CSI is not available at each transmitter.

$a_{k}, b_{k}$ and $c_{k}$, which take values from a set such that $E\left[\left|a_{k}\right|^{2}\right]=E\left[\left|b_{k}\right|^{2}\right]=E\left[\left|c_{k}\right|^{2}\right]=1, k=1,2, \cdots, 8$, represent the modulated signals at transmitter 1 , transmitter 2 and transmitter 3 , respectively. $a_{k}, b_{k}$ and $c_{k}$ are encoded into $2 \times 2$ space-time codeword, denoted by $\boldsymbol{A}_{i}, \boldsymbol{B}_{i}$ and $\boldsymbol{C}_{i}$, respectively, $i=1,2 . \boldsymbol{A}_{i}, \boldsymbol{B}_{i}$ and $\boldsymbol{C}_{i}$ are expressed as

$$
\begin{aligned}
& \boldsymbol{A}_{i}=\left[\begin{array}{cc}
a_{4 i-3}+\mathrm{e}^{j \theta} a_{4 i-1} & -a_{4 i-2}^{*}-\mathrm{e}^{-j \theta} a_{4 i}^{*} \\
a_{4 i-2}+\mathrm{e}^{j \theta} a_{4 i} & a_{4 i-3}^{*}+\mathrm{e}^{-j \theta} a_{4 i-1}^{*}
\end{array}\right] \\
& \boldsymbol{B}_{i}=\left[\begin{array}{cc}
b_{4 i-3}+\mathrm{e}^{j \theta} b_{4 i-1} & -b_{4 i-2}^{*}-\mathrm{e}^{-j \theta} b_{4 i}^{*} \\
b_{4 i-2}+\mathrm{e}^{j \theta} b_{4 i} & b_{4 i-3}^{*}+\mathrm{e}^{-j \theta} b_{4 i-1}^{*}
\end{array}\right] \\
& \boldsymbol{C}_{i}=\left[\begin{array}{cc}
c_{4 i-3}+\mathrm{e}^{j \theta} c_{4 i-1} & -c_{4 i-2}^{*}-\mathrm{e}^{-j \theta} c_{4 i}^{*} \\
c_{4 i-2}+\mathrm{e}^{j \theta} c_{4 i} & c_{4 i-3}^{*}+\mathrm{e}^{-j \theta} c_{4 i-1}^{*}
\end{array}\right]
\end{aligned}
$$

where, $j=\sqrt{-1}, 0<\theta<2 \pi$. The elements of $\boldsymbol{A}_{i}$, the elements of $\boldsymbol{B}_{i}$ and the elements of $\boldsymbol{C}_{i}$ are nonzero by the proper value of $\theta$. Each element of $\boldsymbol{A}_{i}$, each element of $\boldsymbol{B}_{i}$ and each element of $\boldsymbol{C}_{i}$ includes two independent symbols, with the purpose of improving transmission efficiency. If we see the elements of $\boldsymbol{A}_{i}$, the elements of $\boldsymbol{B}_{i}$ and the elements of $\boldsymbol{C}_{i}$ as the modulated signals, $i=1,2, \boldsymbol{A}_{i}, \boldsymbol{B}_{i}$ and $\boldsymbol{C}_{i}$ have an Alamouti structure. The motive for such design is that the effective channel matrix of Alamouti code has orthogonal columns, which can be used to reduce decoding complexity.

At transmitter $1, \boldsymbol{A}_{1}$ and $\boldsymbol{A}_{2}$ are combined according to a certain rule to get codeword $\boldsymbol{A}$. Similarly, $\boldsymbol{B}_{1}$ and $\boldsymbol{B}_{2}$ are combined to obtain codeword $\boldsymbol{B}$ while $\boldsymbol{C}_{1}$ and $\boldsymbol{C}_{2}$ are combined to obtain codeword $\boldsymbol{C}, \boldsymbol{A}, \boldsymbol{B}$ and $\boldsymbol{C}$ are given by

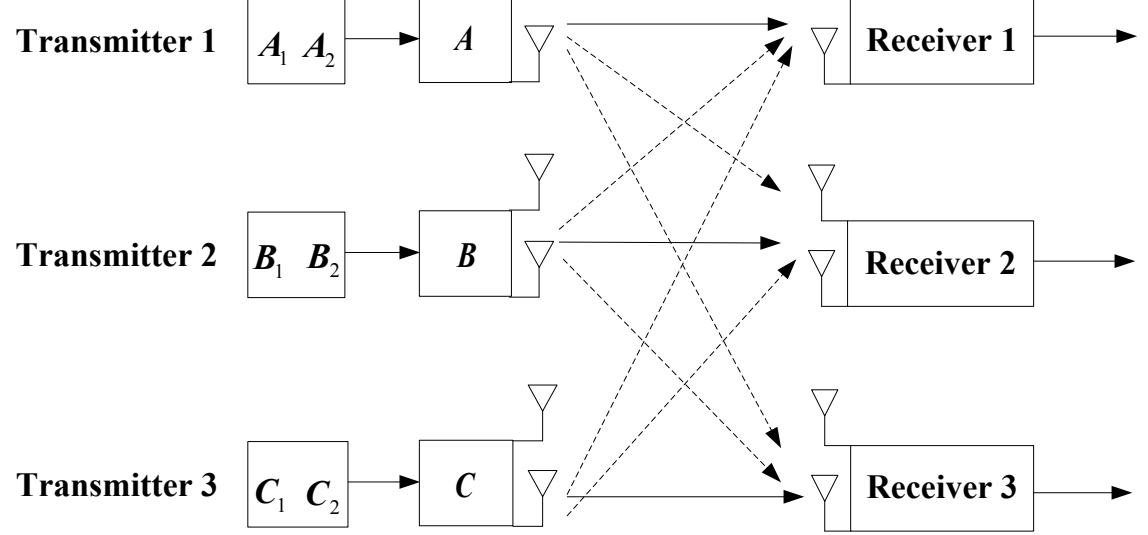

Figure 1. The system model of the proposed scheme. 


$$
\boldsymbol{A}=\left[\begin{array}{ll}
\boldsymbol{A}_{1} & \mathbf{0}_{2} \\
\boldsymbol{A}_{2} & \mathbf{0}_{2}
\end{array}\right], \boldsymbol{B}=\left[\begin{array}{ll}
\boldsymbol{B}_{1} & \mathbf{0}_{2} \\
\mathbf{0}_{2} & \boldsymbol{B}_{2}
\end{array}\right], \boldsymbol{C}=\left[\begin{array}{ll}
\mathbf{0}_{2} & \boldsymbol{C}_{1} \\
\mathbf{0}_{2} & \boldsymbol{C}_{2}
\end{array}\right]
$$

where, $\mathbf{0}_{2}$ represents $2 \times 2$ zero matrix. $\mathbf{0}_{2}$ is introduced because it can be used to mitigate partial multi-user interference, as shown later. $\boldsymbol{A}, \boldsymbol{B}$ and $\boldsymbol{C}$ are the desired codewords for the three receivers, respectively.

As shown in Figure 1, three transmitters send $\boldsymbol{A}, \boldsymbol{B}$ and $\boldsymbol{C}$ on the same frequency band at the same time. The received signals at receiver $i$, denoted by $\boldsymbol{Y}_{i}$, are expressed as

$$
\begin{aligned}
& \boldsymbol{Y}_{1}=\boldsymbol{H}_{11} \boldsymbol{A}+\boldsymbol{H}_{12} \boldsymbol{B}+\boldsymbol{H}_{13} \boldsymbol{C}+\boldsymbol{N}_{1} \\
& \boldsymbol{Y}_{2}=\boldsymbol{H}_{21} \boldsymbol{A}+\boldsymbol{H}_{22} \boldsymbol{B}+\boldsymbol{H}_{23} \boldsymbol{C}+\boldsymbol{N}_{2} \\
& \boldsymbol{Y}_{3}=\boldsymbol{H}_{31} \boldsymbol{A}+\boldsymbol{H}_{32} \boldsymbol{B}+\boldsymbol{H}_{33} \boldsymbol{C}+\boldsymbol{N}_{3}
\end{aligned}
$$

where, $i=1,2,3, \boldsymbol{H}_{i k}$ is the channel from transmitter $k$ to receiver $i$ and $\boldsymbol{N}_{i}$ is the complex additive white Gaussian noise at receiver $i$. The dimension of $\boldsymbol{H}_{i k}$ and $\boldsymbol{N}_{i}$ are both $4 \times 4$. To avoid degenerate channel conditions, we assume that the channel coefficient values are drawn i.i.d. from a continuous distribution and the absolute value of all the channel coefficients is bounded between a nonzero minimum value and a finite maximum value.

The term $\boldsymbol{H}_{11} \boldsymbol{A}$ in Equation (3) is the desired signals for receiver 1 and the term $\boldsymbol{H}_{12} \boldsymbol{B}+\boldsymbol{H}_{13} \boldsymbol{C}$ in Equation (3) is the interference signals for receiver 1. The term $\boldsymbol{H}_{22} \boldsymbol{B}$ in Equation (4) is the desired signals for receiver 2 and the term $\boldsymbol{H}_{21} \boldsymbol{A}+\boldsymbol{H}_{23} \boldsymbol{C}$ in Equation (4) is the interference signals for receiver 2. The term $\boldsymbol{H}_{33} \boldsymbol{C}$ in Equation (5) is the desired signals for receiver 3 and the term $\boldsymbol{H}_{31} \boldsymbol{A}+\boldsymbol{H}_{32} \boldsymbol{B}$ in Equation (5) is the interference signals for receiver 3.

\section{Interference Cancellation Method}

We describe the interference cancellation method at $R_{1}$. It can be similarly used to mitigate interference at $R_{2}$ and to mitigate interference $R_{3}$.

\subsection{Remove the Undesired Codeword}

Let $\quad \boldsymbol{H}_{11}=\left[\begin{array}{ll}\boldsymbol{H}_{1} & \boldsymbol{H}_{2}\end{array}\right], \quad \boldsymbol{H}_{12}=\left[\begin{array}{ll}\boldsymbol{H}_{3} & \boldsymbol{H}_{4}\end{array}\right], \quad \boldsymbol{H}_{13}=\left[\begin{array}{ll}\boldsymbol{H}_{5} & \boldsymbol{H}_{6}\end{array}\right], \quad \boldsymbol{Y}_{1}=\left[\begin{array}{ll}\boldsymbol{Y}_{11} & \boldsymbol{Y}_{21}\end{array}\right]$ and $\boldsymbol{N}_{1}=\left[\begin{array}{ll}\boldsymbol{N}_{11} & \boldsymbol{N}_{21}\end{array}\right]$. The dimension of $\boldsymbol{H}_{l}, \boldsymbol{N}_{i 1}$ and $\boldsymbol{Y}_{i 1}$ are both $4 \times 2$, $l=1,2, \cdots, 6, \quad i=1,2$. Then, (3) can be expressed as

$$
\left[\begin{array}{ll}
\boldsymbol{Y}_{11} & \boldsymbol{Y}_{21}
\end{array}\right]=\left[\begin{array}{ll}
\boldsymbol{H}_{1} \boldsymbol{A}_{1}+\boldsymbol{H}_{2} \boldsymbol{A}_{2}+\boldsymbol{H}_{3} \boldsymbol{B}_{1} & \boldsymbol{H}_{4} B_{2}+\boldsymbol{H}_{5} \boldsymbol{C}_{1}+\boldsymbol{H}_{6} \boldsymbol{C}_{2}
\end{array}\right]+\left[\begin{array}{ll}
\boldsymbol{N}_{11} & \boldsymbol{N}_{21}
\end{array}\right]
$$

(6) is equivalent to the following two equations,

$$
\begin{gathered}
\boldsymbol{Y}_{11}=\boldsymbol{H}_{1} \boldsymbol{A}_{1}+\boldsymbol{H}_{2} \boldsymbol{A}_{2}+\boldsymbol{H}_{3} \boldsymbol{B}_{1}+\boldsymbol{N}_{11} \\
\boldsymbol{Y}_{21}=\boldsymbol{H}_{4} \boldsymbol{B}_{2}+\boldsymbol{H}_{5} \boldsymbol{C}_{1}+\boldsymbol{H}_{6} \boldsymbol{C}_{2}+\boldsymbol{N}_{21}
\end{gathered}
$$

The codeword of transmitter 1 and the partial codeword of transmitter 2 are encompassed by $\boldsymbol{Y}_{11}$. The codeword of transmitter 3 and the partial codeword of transmitter 2 are encompassed by $\boldsymbol{Y}_{21}$. So, the number of interfering codeword is reduced by the introduction of zero matrices. However, both the desired codeword for receiver 1 and the undesired codeword for receiver 1 in terms of 
$\boldsymbol{B}_{1}$ are included by $\boldsymbol{Y}_{11}$. In what follows, we represent how to cancel the undesired codeword $\boldsymbol{B}_{1}$.

Let $\boldsymbol{H}_{1}=\left[\begin{array}{ll}h_{11}^{1} & h_{12}^{1} \\ h_{21}^{1} & h_{22}^{1} \\ h_{31}^{1} & h_{32}^{1} \\ h_{41}^{1} & h_{42}^{1}\end{array}\right], \quad \boldsymbol{H}_{2}=\left[\begin{array}{ll}h_{11}^{2} & h_{12}^{2} \\ h_{21}^{2} & h_{22}^{2} \\ h_{31}^{2} & h_{32}^{2} \\ h_{41}^{2} & h_{42}^{2}\end{array}\right]$ and $\boldsymbol{H}_{3}=\left[\begin{array}{cc}h_{11}^{3} & h_{12}^{3} \\ h_{21}^{3} & h_{22}^{3} \\ h_{31}^{3} & h_{32}^{3} \\ h_{41}^{3} & h_{42}^{3}\end{array}\right]$. Use $y_{i j} \quad$ and $n_{i j}$ to denote the element of $\boldsymbol{Y}_{11}$ and $\boldsymbol{N}_{11}, i=1,2,3,4, j=1,2$. The elements of $\boldsymbol{Y}_{11}$ can be effectively written as

$$
\begin{aligned}
& \boldsymbol{y}_{1}=\boldsymbol{G}_{11}\left[\begin{array}{l}
a_{1}+\mathrm{e}^{j \theta} a_{3} \\
a_{2}+\mathrm{e}^{j \theta} a_{4}
\end{array}\right]+\boldsymbol{G}_{12}\left[\begin{array}{l}
a_{5}+\mathrm{e}^{j \theta} a_{7} \\
a_{6}+\mathrm{e}^{j \theta} a_{8}
\end{array}\right]+\boldsymbol{G}_{13}\left[\begin{array}{l}
b_{1}+\mathrm{e}^{j \theta} b_{3} \\
b_{2}+\mathrm{e}^{j \theta} b_{4}
\end{array}\right]+\boldsymbol{n}_{1} \\
& \boldsymbol{y}_{2}=\boldsymbol{G}_{21}\left[\begin{array}{l}
a_{1}+\mathrm{e}^{j \theta} a_{3} \\
a_{2}+\mathrm{e}^{j \theta} a_{4}
\end{array}\right]+\boldsymbol{G}_{22}\left[\begin{array}{l}
a_{5}+\mathrm{e}^{j \theta} a_{7} \\
a_{6}+\mathrm{e}^{j \theta} a_{8}
\end{array}\right]+\boldsymbol{G}_{23}\left[\begin{array}{l}
b_{1}+\mathrm{e}^{j \theta} b_{3} \\
b_{2}+\mathrm{e}^{j \theta} b_{4}
\end{array}\right]+\boldsymbol{n}_{2} \\
& \boldsymbol{y}_{3}=\boldsymbol{G}_{31}\left[\begin{array}{l}
a_{1}+\mathrm{e}^{j \theta} a_{3} \\
a_{2}+\mathrm{e}^{j \theta} a_{4}
\end{array}\right]+\boldsymbol{G}_{32}\left[\begin{array}{l}
a_{5}+\mathrm{e}^{j \theta} a_{7} \\
a_{6}+\mathrm{e}^{j \theta} a_{8}
\end{array}\right]+\boldsymbol{G}_{33}\left[\begin{array}{l}
b_{1}+\mathrm{e}^{j \theta} b_{3} \\
b_{2}+\mathrm{e}^{j \theta} b_{4}
\end{array}\right]+\boldsymbol{n}_{3} \\
& \boldsymbol{y}_{4}=\boldsymbol{G}_{41}\left[\begin{array}{l}
a_{1}+\mathrm{e}^{j \theta} a_{3} \\
a_{2}+\mathrm{e}^{j \theta} a_{4}
\end{array}\right]+\boldsymbol{G}_{42}\left[\begin{array}{l}
a_{5}+\mathrm{e}^{j \theta} a_{7} \\
a_{6}+\mathrm{e}^{j \theta} a_{8}
\end{array}\right]+\boldsymbol{G}_{43}\left[\begin{array}{l}
b_{1}+\mathrm{e}^{j \theta} b_{3} \\
b_{2}+\mathrm{e}^{j \theta} b_{4}
\end{array}\right]+\boldsymbol{n}_{4}
\end{aligned}
$$

where, $\boldsymbol{G}_{i j}=\left[\begin{array}{cc}h_{i 1}^{j} & h_{i 2}^{j} \\ h_{i 2}^{j *} & -h_{i 1}^{j *}\end{array}\right], \quad \boldsymbol{y}_{i}=\left[\begin{array}{c}y_{i 1} \\ y_{i 2}^{*}\end{array}\right], \quad \boldsymbol{n}_{i}=\left[\begin{array}{c}n_{i 1} \\ n_{i 2}^{*}\end{array}\right], \quad i=1,2,3,4, \quad j=1,2,3 . \boldsymbol{y}_{i}$ and $\boldsymbol{n}_{i}$ are the effective received signals and the effective noise at the $i$-th antenna, respectively. $\left[\begin{array}{l}a_{1}+\mathrm{e}^{j \theta} a_{3} \\ a_{2}+\mathrm{e}^{j \theta} a_{4}\end{array}\right],\left[\begin{array}{l}a_{5}+\mathrm{e}^{j \theta} a_{7} \\ a_{6}+\mathrm{e}^{j \theta} a_{8}\end{array}\right]$ and $\left[\begin{array}{c}b_{1}+\mathrm{e}^{j \theta} b_{3} \\ b_{2}+\mathrm{e}^{j \theta} b_{4}\end{array}\right]$ are the effective transmitted signals of the three transmitters. $\boldsymbol{G}_{i j}$ is the effective channel matrix corresponding to the effective transmitted signals, $i=1,2,3,4$, $j=1,2,3 . \quad \boldsymbol{G}_{i j}$, having orthogonal columns, satisfies $\frac{\boldsymbol{G}_{i j}^{H} \boldsymbol{G}_{i j}}{\left\|\boldsymbol{G}_{i j}\right\|^{2}}=\boldsymbol{I}_{2}$, where $\boldsymbol{I}_{2}$ is unit matrix with dimension $2 \times 2$. The third terms at the right side of Equation (9-12) are the interference signals, which can be mitigated using interference cancellation method as follows

$$
\begin{gathered}
\boldsymbol{z}_{1}=\frac{\boldsymbol{G}_{13}^{H} \boldsymbol{y}_{1}}{\left\|\boldsymbol{G}_{13}\right\|^{2}}-\frac{\boldsymbol{G}_{23}^{H} \boldsymbol{y}_{2}}{\left\|\boldsymbol{G}_{23}\right\|^{2}}=\boldsymbol{P}_{1}\left[\begin{array}{l}
a_{1}+\mathrm{e}^{j \theta} a_{3} \\
a_{2}+\mathrm{e}^{j \theta} a_{4}
\end{array}\right]+\boldsymbol{P}_{2}\left[\begin{array}{l}
a_{5}+\mathrm{e}^{j \theta} a_{7} \\
a_{6}+\mathrm{e}^{j \theta} a_{8}
\end{array}\right]+\boldsymbol{w}_{1} \\
\boldsymbol{z}_{2}=\frac{\boldsymbol{G}_{33}^{H} \boldsymbol{y}_{3}}{\left\|\boldsymbol{G}_{33}\right\|^{2}}-\frac{\boldsymbol{G}_{43}^{H} \boldsymbol{y}_{4}}{\left\|\boldsymbol{G}_{43}\right\|^{2}}=\boldsymbol{P}_{3}\left[\begin{array}{l}
a_{1}+\mathrm{e}^{j \theta} a_{3} \\
a_{2}+\mathrm{e}^{j \theta} a_{4}
\end{array}\right]+\boldsymbol{P}_{4}\left[\begin{array}{l}
a_{5}+\mathrm{e}^{j \theta} a_{7} \\
a_{6}+\mathrm{e}^{j \theta} a_{8}
\end{array}\right]+\boldsymbol{w}_{2}
\end{gathered}
$$

where, $\quad \boldsymbol{P}_{i}=\frac{\boldsymbol{G}_{13}^{H} \boldsymbol{G}_{1 i}}{\left\|\boldsymbol{G}_{13}\right\|^{2}}-\frac{\boldsymbol{G}_{23}^{H} \boldsymbol{G}_{2 i}}{\left\|\boldsymbol{G}_{23}\right\|^{2}}, \quad \boldsymbol{P}_{j}=\frac{\boldsymbol{G}_{33}^{H} \boldsymbol{G}_{3(j-2)}}{\left\|\boldsymbol{G}_{33}\right\|^{2}}-\frac{\boldsymbol{G}_{43}^{H} \boldsymbol{G}_{4(j-2)}}{\left\|\boldsymbol{G}_{43}\right\|^{2}}$

$\boldsymbol{w}_{1}=\frac{\boldsymbol{G}_{13}^{H} \boldsymbol{n}_{1}}{\left\|\boldsymbol{G}_{13}\right\|^{2}}-\frac{\boldsymbol{G}_{23}^{H} \boldsymbol{n}_{2}}{\left\|\boldsymbol{G}_{23}\right\|^{2}}, \quad \boldsymbol{w}_{2}=\frac{\boldsymbol{G}_{33}^{H} \boldsymbol{n}_{3}}{\left\|\boldsymbol{G}_{33}\right\|^{2}}-\frac{\boldsymbol{G}_{43}^{H} \boldsymbol{n}_{4}}{\left\|\boldsymbol{G}_{43}\right\|^{2}}, \quad i=1,2, j=1,2 . \boldsymbol{z}_{i}$ and $\boldsymbol{w}_{i}$ are the signals and the noise after linear operation, respectively, $i=1,2 . \quad \boldsymbol{P}_{i}$ is effective channel matrix after linear operation, $i=1,2,3,4$. The dimension of $\boldsymbol{z}_{i}$ and the dimension $\boldsymbol{w}_{i}$ are both $2 \times 1, i=1,2$. The dimension of $\boldsymbol{P}_{i}$ is $2 \times 2$, 
$i=1,2,3,4$. Symbols transmitted from transmitter 2 and symbols transmitted from transmitter 3 are not included in (13-14). So multi-user interference is mitigated through linear operation on the received signals.

\subsection{Separate the Desired Codewords}

Even if multi-user interference is cancelled, the desired codeword at receiver 1 includes 8 independent modulated symbols, namely $a_{1}, a_{2}, \cdots, a_{8}$, as shown in (13-14). If we decode $a_{1}, a_{2}, \cdots, a_{8}$ based on (13-14), the complexity of maximum likelihood $(\mathrm{ML})$ algorithm is proportional to the 8 th power of modulation order, which is extremely high. So, it is necessary to divide these symbols into several groups. Take operation on $z_{1}$ and $z_{2}$ to get,

$$
\begin{gathered}
\boldsymbol{x}_{1}=\frac{\boldsymbol{P}_{2}^{H} \boldsymbol{z}_{1}}{\left\|\boldsymbol{P}_{2}\right\|^{2}}-\frac{\boldsymbol{P}_{4}^{H} \boldsymbol{z}_{2}}{\left\|\boldsymbol{P}_{4}\right\|^{2}}=\boldsymbol{Q}_{1}\left[\begin{array}{c}
a_{1}+\mathrm{e}^{j \theta} a_{3} \\
a_{2}+\mathrm{e}^{j \theta} a_{4}
\end{array}\right]+\boldsymbol{w}_{3} \\
\boldsymbol{x}_{2}=\frac{\boldsymbol{P}_{1}^{H} \boldsymbol{z}_{1}}{\left\|\boldsymbol{P}_{1}\right\|^{2}}-\frac{\boldsymbol{P}_{3}^{H} \boldsymbol{z}_{2}}{\left\|\boldsymbol{P}_{3}\right\|^{2}}=\boldsymbol{Q}_{2}\left[\begin{array}{l}
a_{5}+\mathrm{e}^{j \theta} a_{7} \\
a_{6}+\mathrm{e}^{j \theta} a_{8}
\end{array}\right]+\boldsymbol{w}_{4}
\end{gathered}
$$

where, $\boldsymbol{Q}_{1}=\frac{\boldsymbol{P}_{2}^{H} \boldsymbol{P}_{1}}{\left\|\boldsymbol{P}_{2}\right\|^{2}}-\frac{\boldsymbol{P}_{4}^{H} \boldsymbol{P}_{3}}{\left\|\boldsymbol{P}_{4}\right\|^{2}}, \quad \boldsymbol{Q}_{2}=\frac{\boldsymbol{P}_{1}^{H} \boldsymbol{P}_{2}}{\left\|\boldsymbol{P}_{1}\right\|^{2}}-\frac{\boldsymbol{P}_{3}^{H} \boldsymbol{P}_{4}}{\left\|\boldsymbol{P}_{3}\right\|^{2}}, \quad \boldsymbol{w}_{3}=\frac{\boldsymbol{P}_{2}^{H} \boldsymbol{w}_{1}}{\left\|\boldsymbol{P}_{2}\right\|^{2}}-\frac{\boldsymbol{P}_{4}^{H} \boldsymbol{w}_{2}}{\left\|\boldsymbol{P}_{4}\right\|^{2}}$, $\boldsymbol{w}_{4}=\frac{\boldsymbol{P}_{1}^{H} \boldsymbol{w}_{1}}{\left\|\boldsymbol{P}_{1}\right\|^{2}}-\frac{\boldsymbol{P}_{3}^{H} \boldsymbol{w}_{2}}{\left\|\boldsymbol{P}_{3}\right\|^{2}} \cdot a_{1}, a_{2}, a_{3}$ and $a_{4}$ are encompassed by $\boldsymbol{A}_{1}$ whereas $a_{5}, a_{6}, a_{7}$ and $a_{8}$ are encompassed by $\boldsymbol{A}_{2}$. So, the elements of $\boldsymbol{A}_{1}$ and the elements of $\boldsymbol{A}_{2}$ are separated, as shown in (15-16). The decoding complexity can be reduced.

From the above analysis, not only multi-user interference is cancelled, but also the two desired codewords $A_{1}$ and $A_{2}$ are separated. Similar operation can be performed at the other two receivers. Note that neither feedback information nor the unidirectional cooperative link is required.

\section{Decoding Method}

In this section, we will show how to decode, taking receiver 1 as example.

$\boldsymbol{G}_{i j}$ has an Alamouti structure, $i=1,2,3,4, j=1,2,3$. Due to the completeness of matrix addition, matrix multiplication, and the scalar multiplication of the Alamouti matrix, $\boldsymbol{Q}_{1}$ and $\boldsymbol{Q}_{2}$ still have the Alamouti structure. Let $\boldsymbol{Q}_{1}=\left[\begin{array}{cc}q_{1} & -q_{2}^{*} \\ q_{2} & q_{1}^{*}\end{array}\right]$ and $\boldsymbol{Q}_{2}=\left[\begin{array}{cc}q_{3} & -q_{4}^{*} \\ q_{4} & q_{3}^{*}\end{array}\right]$. Multiply both sides of (15) with $\boldsymbol{Q}_{1}^{H}$ to get

$$
\boldsymbol{Q}_{1}^{H} \boldsymbol{x}_{1}=\left[\begin{array}{l}
x_{1} \\
x_{2}
\end{array}\right]=\left[\begin{array}{cc}
\left|q_{1}\right|^{2}+\left|q_{2}\right|^{2} & 0 \\
0 & \left|q_{1}\right|^{2}+\left|q_{2}\right|^{2}
\end{array}\right]\left[\begin{array}{l}
a_{1}+\mathrm{e}^{j \theta} a_{3} \\
a_{2}+\mathrm{e}^{j \theta} a_{4}
\end{array}\right]+\boldsymbol{Q}_{1}^{H} \boldsymbol{w}_{3}
$$

Multiply both sides of (16) with $\boldsymbol{Q}_{2}^{H}$ to get

$$
\boldsymbol{Q}_{2}^{H} \boldsymbol{x}_{2}=\left[\begin{array}{c}
x_{3} \\
x_{4}
\end{array}\right]=\left[\begin{array}{cc}
\left|q_{3}\right|^{2}+\left|q_{4}\right|^{2} & 0 \\
0 & \left|q_{3}\right|^{2}+\left|q_{4}\right|^{2}
\end{array}\right]\left[\begin{array}{l}
a_{5}+\mathrm{e}^{j \theta} a_{7} \\
a_{6}+\mathrm{e}^{j \theta} a_{8}
\end{array}\right]+\boldsymbol{Q}_{2}^{H} \boldsymbol{w}_{4}
$$


In (17), the channel corresponding to $a_{1}+\mathrm{e}^{j \theta} a_{3}$ is orthogonal to the channel corresponding to $a_{2}+\mathrm{e}^{j \theta} a_{4}$. So we can decode $a_{1}+\mathrm{e}^{j \theta} a_{3}$ and $a_{2}+\mathrm{e}^{j \theta} a_{4}$ separately. Similarly, we can decode $a_{5}+\mathrm{e}^{j \theta} a_{7}$ and $a_{6}+\mathrm{e}^{j \theta} a_{8}$ separately as well. According to the analysis mentioned above, the decoding method is as follows.

Step1, obtain $\boldsymbol{G}_{11}, \boldsymbol{G}_{12}, \cdots, \boldsymbol{G}_{43}$ from the channel matrices, and then compute $\boldsymbol{P}_{1}, \boldsymbol{P}_{2}, \boldsymbol{P}_{3}$ and $\boldsymbol{P}_{4}$ from $\boldsymbol{G}_{11}, \boldsymbol{G}_{12}, \cdots, \boldsymbol{G}_{43}$;

Step 2, compute $\boldsymbol{Q}_{1}=\frac{\boldsymbol{P}_{2}^{H} \boldsymbol{P}_{1}}{\left\|\boldsymbol{P}_{2}\right\|^{2}}-\frac{\boldsymbol{P}_{4}^{H} \boldsymbol{P}_{3}}{\left\|\boldsymbol{P}_{4}\right\|^{2}}$ and $\boldsymbol{Q}_{2}=\frac{\boldsymbol{P}_{1}^{H} \boldsymbol{P}_{2}}{\left\|\boldsymbol{P}_{1}\right\|^{2}}-\frac{\boldsymbol{P}_{3}^{H} \boldsymbol{P}_{4}}{\left\|\boldsymbol{P}_{3}\right\|^{2}}$ through direct multiplications and subtraction;

Step 3, obtain $\boldsymbol{y}_{1}, \boldsymbol{y}_{2}, \boldsymbol{y}_{3}$ and $\boldsymbol{y}_{4}$ according to the received signals, as in (9-12);

Step 4, take operation on $\boldsymbol{y}_{1}, \boldsymbol{y}_{2}, \boldsymbol{y}_{3}$ and $\boldsymbol{y}_{4}$ to get $\boldsymbol{z}_{1}$ and $\boldsymbol{z}_{2}$, $z_{1}=\frac{G_{13}^{H} y_{1}}{\left\|G_{13}\right\|^{2}}-\frac{G_{23}^{H} y_{2}}{\left\|G_{23}\right\|^{2}}, \quad z_{2}=\frac{G_{33}^{H} y_{3}}{\left\|G_{33}\right\|^{2}}-\frac{G_{43}^{H} y_{4}}{\left\|G_{43}\right\|^{2}} ;$

Step 5, take operation on $\boldsymbol{z}_{1}$ and $\boldsymbol{z}_{2}$ to get $\boldsymbol{x}_{1}=\frac{\boldsymbol{P}_{2}^{H} \boldsymbol{z}_{1}}{\left\|\boldsymbol{P}_{2}\right\|^{2}}-\frac{\boldsymbol{P}_{4}^{H} \boldsymbol{z}_{2}}{\left\|\boldsymbol{P}_{4}\right\|^{2}}$ and $\boldsymbol{x}_{2}=\frac{\boldsymbol{P}_{1}^{H} \boldsymbol{z}_{1}}{\left\|\boldsymbol{P}_{1}\right\|^{2}}-\frac{\boldsymbol{P}_{3}^{H} \boldsymbol{z}_{2}}{\left\|\boldsymbol{P}_{3}\right\|^{2}}$, furthermore, computer $\left[\begin{array}{c}x_{1} \\ x_{2}\end{array}\right]=\boldsymbol{Q}_{1}^{H} \boldsymbol{x}_{1}$ and $\left[\begin{array}{c}x_{3} \\ x_{4}\end{array}\right]=\boldsymbol{Q}_{2}^{H} \boldsymbol{x}_{2}$ through direct multiplications;

Step 6, estimate $a_{1}$ and $a_{3}$ by $\left(\hat{a}_{1}, \hat{a}_{3}\right)=\underbrace{\arg \min }_{a_{1}, a_{3}}\left[x_{1}-\left(\left|q_{1}\right|^{2}+\left|q_{2}\right|^{2}\right)\left(a_{1}+\mathrm{e}^{j \theta} a_{3}\right)\right]$, where $\hat{a}_{1}$ and $\hat{a}_{3}$ are the estimation of $a_{1}$ and $a_{3}$, respectively;

Step 7, estimate $a_{2}$ and $a_{4}$ by $\left(\hat{a}_{2}, \hat{a}_{4}\right)=\underbrace{\arg \min }_{a_{2}, a_{4}}\left[x_{2}-\left(\left|q_{1}\right|^{2}+\left|q_{2}\right|^{2}\right)\left(a_{2}+\mathrm{e}^{j \theta} a_{4}\right)\right]$, where $\hat{a}_{2}$ and $\hat{a}_{4}$ are the estimation of $a_{2}$ and $a_{4}$, respectively;

Step 8, estimate $a_{5}$ and $a_{6}$ by $\left(\hat{a}_{5}, \hat{a}_{6}\right)=\underbrace{\arg \min }_{a_{5}, a_{6}}\left[x_{3}-\left(\left|q_{1}\right|^{2}+\left|q_{2}\right|^{2}\right)\left(a_{5}+\mathrm{e}^{j \theta} a_{6}\right)\right]$, where $\hat{a}_{5}$ and $\hat{a}_{6}$ are the estimation of $a_{5}$ and $a_{6}$, respectively;

Step 9, estimate $a_{7}$ and $a_{8}$ by $\left(\hat{a}_{7}, \hat{a}_{8}\right)=\underbrace{\arg \min }_{a_{7}, a_{8}}\left[x_{4}-\left(\left|q_{1}\right|^{2}+\left|q_{2}\right|^{2}\right)\left(a_{7}+\mathrm{e}^{j \theta} a_{8}\right)\right]$, where $\hat{a}_{7}$ and $\hat{a}_{8}$ are the estimation of $a_{7}$ and $a_{8}$, respectively;

The ML decoding complexity in step (6-9) is proportional to the square of modulation order.

It is easy to see from (17) that the channel corresponding to $a_{1}+\mathrm{e}^{j \theta} a_{3}$ is $\left|q_{1}\right|^{2}+\left|q_{2}\right|^{2}$, which includes two independent path with gain $\left|q_{1}\right|^{2}$ and $\left|q_{2}\right|^{2}$. So $a_{1}+\mathrm{e}^{j \theta} a_{3}$ has a diversity gain of two, which is equal to that of [11]. Similar arguments can be used to prove that other symbols achieve a diversity of two as well. 


\section{Simulation Results}

6 independent modulated signals are sent over two time slots in [11]. Feedback information, namely 16 complex numbers, are required at the transmitters, which occupy $16 / 3$ time slot if these three receivers adopt the same transmission and the same code mode as the transmitters. So, 22/3 time slot is required to send 6 independent modulated signals in [11]. The transmission efficiency is $3 / 22$ symbol per antenna per time slot. 24 independent modulated signals are sent over four time slots in the proposed scheme. The transmission efficiency is $1 / 2$ symbol per antenna per time slot, which is $11 / 3$ times as that of [11].

Performance of the proposed scheme and performance of the scheme in [11] are compared in Table 1 . Two schemes have the same diversity gain. The advantage of the proposed scheme is that neither feedback information nor unidirectional cooperative link is required, and the transmission efficiency is improved. The disadvantage is that the decoding complexity is proportional to the square of modulation order, higher than that of [11], which is proportional to the modulation order.

In Figure 2, we simulate the average BER curves of the proposed scheme and the scheme in [11]. We consider uncoded systems, in which all the underlying links experience Rayleigh fading channels. The data symbols are assumed to be 4QAM symbols and 16QAM symbols, generated independently at each transmitter. The average BER is determined through monte-carlo simulations. The

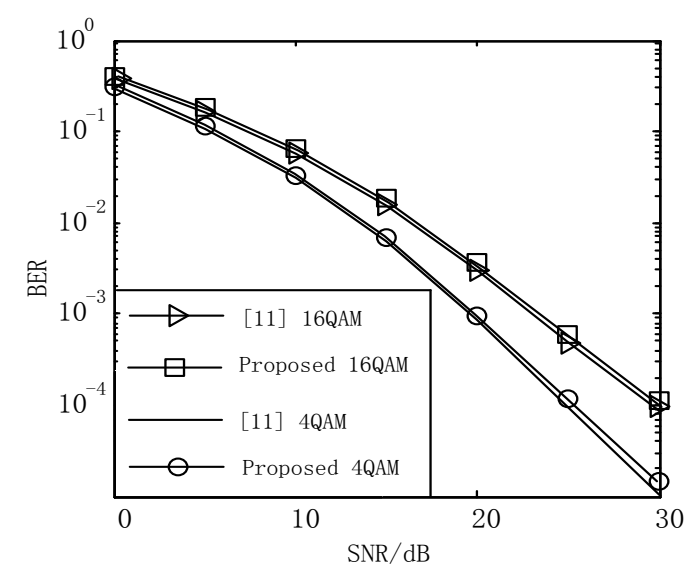

Figure 2. The BER curves of the two schemes.

Table 1. Performance of the two schemes.

\begin{tabular}{ccc}
\hline Scheme & Proposed & Ref. [11] \\
\hline Transmission efficiency & $1 / 2$ symbol/antenna/time slot & $3 / 22$ symbol/antenna/time slot \\
Diversity gain & 2 & 2 \\
Feedback & No & Local CSI \\
Unidirectional cooperative link & No & Transmit two complex number \\
Decoding complexity & $\begin{array}{c}\text { Proportional to the square } \\
\text { of modulation order }\end{array}$ & Proportional to the modulation order \\
\hline
\end{tabular}


slope of BER curves represents diversity gain, which is 2 in these two schemes. It can be seen from this figure that the slope of BER curves of the proposed scheme is almost the same as that of [11], which demonstrate the validity of theoretical analysis.

\section{Conclusion}

In this paper, the multi-user interference is cancelled by the design of the codeword and linear operation on the received signals. Compared with the existing scheme for the same scene, the proposed scheme doesn't require feedback information and doesn't require unidirectional cooperative link, while keeping the same diversity gain. However, the scheme is limited to three transmitter-receiver pairs, where each node is equipped with four antennas. Future work on this scheme includes extending the application scene.

\section{Acknowledgements}

The authors would like to thank the reviewers for their detailed reviews and constructive comments, which have helped improve the quality of this paper. This work was supported in part by National Natural Science Foundation of China under Grant No. 51174263.

\section{References}

[1] Gesbert, D., Shafi, M. and Shiu, D. (2013) From Theory to Practice: An Overview of Mimo Space-Time Coded Wireless Systems. IEEE Journal on Selected Areas in Communications, 21, 281-302. https://doi.org/10.1109/JSAC.2003.809458

[2] Chen, X., Song, S.H. and Letaief, K.B. (2014) Interference Alignment in Dual-Hop MIMO Interference Channel. IEEE Transactions on Wireless Communications, 13, 1274-1283. https://doi.org/10.1109/TWC.2014.012114.121952

[3] Lu, L. and Zhang, W. (2014) Blind Interference Alignment with Diversity in K-User Interference Channels. IEEE Transactions on Communication, 62, 2850-2859. https://doi.org/10.1109/TCOMM.2014.2333516

[4] Maddah-Ali, M., Motahari, A. and Khandani, A. (2008) Communication over MIMO X Channels: Interference Alignment, Decomposition, and Performance Analysis. IEEE Transactions on Information Theory, 54, 3457-3470. https://doi.org/10.1109/TIT.2008.926460

[5] Xie, B., Li, Y., Minn, H. and Nosratinia, A. (2013) Adaptive Interference Alignment with CSI Uncertainty. IEEE Transactions on Communications, 61, 792-801. https://doi.org/10.1109/TCOMM.2012.121112.110589

[6] Chen, X., Song, S.H. and Letaief, K.B. (2014) Interference Alignment in Dual-Hop MIMO Interference Channel. IEEE Transactions on Wireless Communications, 13, 1274-1283. https://doi.org/10.1109/TWC.2014.012114.121952

[7] Ganesan, A. and Rajan, B.S. (2013) Interference Alignment with Diversity for the $2 \times 2$ X Network with Three Antennas. arXiv:1304.1432[cs.IT]

[8] Shi, L., Zhang, W. and Xia, X.G. (2013) Space-Time Block Code Designs for Two-User MIMO X Channels. IEEE Transactions on Communications, 61, 38063815. https://doi.org/10.1109/TCOMM.2013.080613.120972

[9] Li, L.B. and Jafarkhani, H. (2013) Maximum-Rate Transmission with Improved Di- 
versity Gain for Interference Networks. IEEE Transactions on Information Theory, 59, 5313-5330. https://doi.org/10.1109/TIT.2013.2264479

[10] Ganesan, A. and Rajan, B.S. (2014) Interference Alignment with Diversity for the 2 $\times 2 \mathrm{X}$ Network with Four Antennas. IEEE Transactions on Information Theory, 60, 3576-3592. https://doi.org/10.1109/TIT.2014.2313614

[11] Zaki, A., Wang, C. and Rasmussen, L.K. (2013) Combining Interference Alignment and Alamouti Codes for the 3-User Mimo Interference Channel. IEEE Wireless Communications and Networking Conference, Shanghai, 3563-3567.

https://doi.org/10.1109/wcnc.2013.6555138

\section{Open Access Library}

Submit or recommend next manuscript to OALib Journal and we will provide best service for you:

- Publication frequency: Monthly

- 9 subject areas of science, technology and medicine

- Fair and rigorous peer-review system

- Fast publication process

- Article promotion in various social networking sites (LinkedIn, Facebook, Twitter, etc.)

- Maximum dissemination of your research work

Submit Your Paper Online: Click Here to Submit

Or Contact service@oalib.com 